\title{
Microsystems for Optical Gas Sensing Incorporating the Solvatochromic Dye Nile Red
}

\author{
Dong Li, Christopher A. Mills and Jonathan M. Cooper* \\ Bioelectronics Research Centre, Department of Electronics \& Electrical Engineering, \\ University of Glasgow, Rankine Building, Oakfield Avenue, Glasgow, G12 8LT, UK. \\ * electronic mail: J.Cooper@elec.gla.ac.uk
}

\begin{abstract}
An optical gas sensor has been developed based on the fluorescence emission of the solvatochromic dye, Nile Red, immobilised within various polymers with different physical properties. Microsystems, made either in SU-8/glass or microstructure glass (MSG) substrates, were used to host the environmentally sensitive fluorescent dye within the polymer matrices. The MSG devices have been found to have superior sensitivity to analytes (up to 7 times greater) and recovery times (up to $50 \%$ faster) than analogous structures made in SU-8. Measurement of the fluorescence at two separate wavelengths confirmed the ability of the MSG sensor array to produce a "fingerprint" response for separate analytes with a high degree of repeatability (the standard deviation of the average response to a given analyte was less than $1 \%$ ). Used in conjunction with pattern recognition techniques, the arrays show potential for gas identification and discrimination.
\end{abstract}

Keywords: micro-gas sensor; solvatochromic dye; Nile Red; microstructure glass; polymer matrices 


\section{Introduction}

The field of artificial olfaction and gas determination is one of the fastest growing areas of sensing both commercially and within academic research areas [1-15]. Much of the work in this field to date concentrates on identification and quantification of multiple analyte species by employing miniaturised sensors. Researchers have thus put a large amount of effort into promoting multiplexed sensor arrays that detect a wide range of analytes using a limited number of sensing elements. However there is still a need for further research to produce artificial olfactory systems that have good sensing characteristics, including fast response times, reversibility, and high sensitivities, while having a minimum hardware requirement.

One example of a multiplexed sensor system is the electronic nose. A requirement for sensors within an electronic nose system, as compared to stand-alone gas sensors, is the ability to sense many disparate chemicals (broadband response) with high sensitivity. This would allow identification and discrimination of simple and complex odours, as well as allowing multi-component analysis. One way to achieve this is to have a multitude of specific receptors with high binding coefficients, such as the "smell-seeing" colorimetric sensor array for odour visualisation reported by Rakow and Suslick [14]. Alternatively, an array of less specific sensors may be used, employing a training protocol and pattern recognition technique, in a method similar to that developed by nature in the mammalian olfaction system $[13,16-18]$. Within the context of the application of sensor arrays, ideally every vapour will cause some or all of the sensing elements to respond differently, producing a unique response pattern for each analyte.

Electronic nose systems have been produced using a variety of different sensor types, including surface acoustic wave devices, [1-3] conducting polymers, [4-6] metal oxide semiconductors, [7] carbon black/polymer composite gas sensors, [8-10] and fluorescent microspheres [11-13]. In the work presented here, we have chosen to explore the area of 
optical sensor arrays based upon the use of solvatochromic dyes. Optical-based sensor systems have the advantage over electronics-based systems of being able to provide a large amount of information, which can be readily multiplexed, almost instantly. Remote sensing, using inexpensive instrumentation and without the need for amplifiers, is also possible and the extreme sensitivity of the system will allow in the future for single molecule detection.

Using a detection system similar to that used by Walt et al [17, 18], we have investigated the temporal response and equilibrium fluorescence change of dye/polymer systems. However, rather than using a fibre optic bundle as the substrate for the sensor, the response and recovery times of two detectors, constructed from microstructured glass and SU-8/glass, have been compared. Analytes of varying polarity (methanol, butan-2-ol and hexane) have been tested, using sensors incorporating polymers with increasing hydrophobicity. In contrast to previous studies, in our work the emission spectral shift of individual sensors has been examined by comparing the fluorescence emission of the dye/polymer at two different wavelengths. This is done with a view to producing a discriminatory sensor system for complex vapour mixtures in the future.

\section{Experimental}

\subsection{Materials}

Figure 1 shows the chemical structures of the dye and the polymers used in fabricating the sensors. Nile Red dye (97\%) was purchased from Aldrich Chemical Co, UK. and used as received. Poly(dimethylsiloxane) (PDMS) base and curing agent was supplied by RS Components Ltd, UK. Poly(methylmethacrylate) (PMMA), poly(acrylonitrile-co-butadieneco-styrene) (PABS), Poly(caprolactone) (PC), poly(styrene) (PS), poly(vinylpyrrolidone) (PVP), were purchased from Aldrich. Negative photoresist SU-8(10) was purchased from 
Microchem. Corp. Ltd. (Newton, MA, USA) and contained 59 wt \% solid in $\gamma$-butyrolactate (GBL) solvent. All other chemicals used were AR grade and used as supplied by Aldrich. Nile Red stock solution (0.01 wt \%) was prepared in chloroform. Polymer stock solution was prepared by dissolving $0.04 \mathrm{~g}$ of the selected polymer in $10 \mathrm{ml}$ of chloroform, and ultrasonicating to produce a homogenous solution. Nile Red/polymer solution was prepared prior to their use by mixing $0.1 \mathrm{ml}$ of the Nile Red stock solution with $1.0 \mathrm{ml}$ of the polymer stock solution.

\subsection{Fabrication methods}

\subsubsection{Microstructured glass sensors}

Unetched microstructure glass (MSG) bundles with $50 \mu \mathrm{m}$ channel diameters were purchased from Galileo Electro-Optics Corp. (MA). The bundle was wafered perpendicular to the channel axis into $0.5 \mathrm{~mm}$ thick sections, using a diamond saw. The wafers were then cleaned in methanol to remove surface contamination, and the soft glass core was etched using $2 \mathrm{M} \mathrm{HCl}$. The MSG was then diced into $12 \mathrm{~mm} \times 12 \mathrm{~mm}$ square chips following the method of Beniot et al [19].

The glass used for the microstructure substrate was clear potash lead glass (Corning type 8161) containing 55\% lead oxide and $35 \%$ silicon dioxide. In terms of optical properties, this type of glass is characterized by a low optical absorption coefficient in the visible region of the electromagnetic spectrum, and a high index of refraction (1.7 at 500 $\mathrm{nm})$. Figure 2 shows a SEM micrograph and a cross-sectional diagram of a $50 \mu \mathrm{m}$ channel diameter, $0.5 \mathrm{~mm}$ thick MSG chip. At this level of magnification, individual microchannels can easily be distinguished, and the superstructure ("bundling") that defines the hexagonal packing of the channels is apparent. Patterning of different dye/polymers on to MSG was 
achieved by employing a micromanipulator that was used to immobilise the dye/polymer on the glass surface. In the SEM micrograph the dye/polymer coated area of the MSG is to the bottom right of figure 2, uncoated MSG is to the top left. As can be seen in the figure, the polymer film enters the individual channels of the MSG superstructure and coats the walls of the channel.

\subsubsection{SU-8 sensor microfabrication}

Developed by IBM, SU-8 is a negative tone, epoxy-based resin photoresist for microstructure fabrication with high aspect ratios. SU-8 can be spun to a thickness of between 1-1000 microns using a single coat, thereby reducing costs and increasing throughout. Figure 3(a) shows a SEM micrograph and optical image of the sensor array structure, fabricated from microstructured SU-8(10) patterned on glass, prior to addition of the glass top plate and upon introduction of 8 dye/polymer combinations respectively. Figure 3(b) shows a cross-sectional diagram of the detector.

The microstructure was fabricated in the following way. Glass substrates were cleaned by successive, ultrasonicated immersions in Opticlear (National Diagnostics, USA), acetone, and methanol, followed by an acid clean with Piranha solution $\left(7 \mathrm{H}_{2} \mathrm{SO}_{4} / 1 \mathrm{H}_{2} \mathrm{O}_{2}\right)$. The substrates were then dehydrated at $200^{\circ} \mathrm{C}$ for 1 hour, then allowed to cool to room temperature. SU-8 (10) was spread across the whole substrate, approximately $4 \mathrm{ml}$ of SU-8 being applied per $10 \mathrm{~cm}$-diameter of the substrate to be coated. The SU-8 was spun at 500 rpm for 12 seconds then allowed to stand for 30 minutes on a flat, horizontal plane to produce a uniformly thick film prior to baking. The polymer was then soft-baked at $60^{\circ} \mathrm{C}$ for 15 minutes, followed by 4 hours at $95^{\circ} \mathrm{C}$, then slowly cooled to room temperature. The thin polymer film was exposed with UV light for 50 seconds, through a patterned ferric mask, using a mask aligner with an exposure dose of $20 \mathrm{~mW} \mathrm{~cm}$. The sample was post-baked at 
$60^{\circ} \mathrm{C}$ for 5 minutes, followed by 60 minutes at $95^{\circ} \mathrm{C}$ to complete the cross-linking in the polymer. Again, the sample was slowly cooled to room temperature. The exposed sample was developed using EC-solvent for 180 seconds, rinsed with iso-propanol, and then dried using a nitrogen gas stream.

The baking time depends on the thickness of the SU-8 film desired and, as a consequence, the exposure time depends on the baking time. Cross-linked SU-8 shows excellent chemical resistance when cured above $100^{\circ} \mathrm{C}$, and has good thermal stability at temperatures greater than $200^{\circ} \mathrm{C}$, allowing it to be used in processing techniques such as metal wet etch and reactive ion etching (RIE). SU-8 will also withstand most standard acidic and alkaline plating solutions, including strongly alkaline solutions ( $\mathrm{pH}$ 13) at high temperature, making it an ideal, chemically resistant device substrate. This method was used to produce a device with an SU-8 layer thickness of $200 \mu \mathrm{m}$. Finally, a glass top plate was used to define an area of $0.19 \mathrm{~mm}^{2}$ within the device which contains the sensors.

\subsection{Instrumentation}

The experimental apparatus combined a CCD imaging system and a Zeiss inverted epifluorescence microscope (Axiovert 200). The microscope included a monochromator that defined the wavelength of the excitation radiation used in this work $(533 \mathrm{~nm})$ as well as two emission filters for analysis of the emitted radiation. The filters selected wavelengths of 580 $\mathrm{nm}$ (band-pass $10 \mathrm{~nm}$ ) and $640 \mathrm{~nm}$ (band-pass $10 \mathrm{~nm}$ ) and were mounted within a Lambda 10-2 filter wheel in the light path before the CCD camera. This allowed rapid wavelength selection during the experimental analysis of the emitted fluorescence. A dichroic mirror (single band 555DRLP, Glen Spectra Ltd., UK) was used to divert the excitation beam onto the sensors but allowed the emitted fluorescence to pass through to the imaging CCD camera. The fluorescence was detected using a cooled Imago CCD camera, with the help of 
TILLVision acquisition software (T. I. L. L. Photonics GmbH, Germany). The peltier-cooled camera uses a $1 \frac{1}{2}$ inch interline-transfer-chip containing $640 \times 480$ pixels, with a pixel size of $9.9 \mu \mathrm{m} \times 9.9 \mu \mathrm{m}$, and employed a $100 \mathrm{~ms}$ exposure time. A schematic diagram of the imaging apparatus is shown in Figure 4.

A bespoke gas flow system was constructed which, when controlled by an electronic valve, supplied a reproducible vapor pulse of methanol, butan-2-ol or hexane to the sensors. The gas-tight flow cell, incorporating the sensors, was also fabricated in-house, through which the gas flow rate was $0.4 \mathrm{~L} \mathrm{~min}^{-1}$. Figure 5 shows a schematic diagram of the gas-flow system incorporating a five port electric valve (Omnifit Ltd., UK, No. 11527).

\subsection{Experimental methods}

Three different measurements were made in order to compare the characteristic responses of the optical gas sensors. Firstly, the fluorescent temporal responses were measured upon introducing a 2 second analyte pulse to the sensors (allowing the vapor to then rediffuse out of the system). Measurements of the fluorescence were made at 0.15 second intervals, up to 60 seconds. Secondly, measurements at an equilibrium state were completed where an analyte vapour stream was introduced to the sensors in a continuous stream. The system was allowed to equilibrate for 10-15 seconds before measuring the fluorescence intensity. In both cases, the intensity of the fluorescence was measured using the two emission filters. Finally, the repeatability of the fluorescence measurements was investigated by introducing a 2 second vapour pulse to the sensor array on the MSG device, followed by a 20 second purge with nitrogen. The fluorescent intensity repeatability was measured using an exposure time of $20 \mathrm{~ms}$ and a $200 \mathrm{~ms}$ cycle time, using the $640 \mathrm{~nm}$ filter.

\section{Results}


Figure 6 shows the temporal response curves of two sensors, (a) Nile Red/PDMS and (b) Nile Red/PVP, on a MSG device upon the introduction of a 2 second pulse of analyte gas. The symbols are used to help discriminate between the line plots for each analyte accordingly; (O) butan-2-ol, $(\nabla)$ hexane and $(\square)$ methanol. The $580 \mathrm{~nm}$ filter was used to observe the fluorescence at room temperature. Upon introducing the analytes, the intensity of the fluorescence emitted from the Nile Red/PDMS sensor is seen to increase, with the sensor responding first to the introduction of methanol. Conversely, for the Nile Red/PVP sensor the intensity decreases and the introduction of hexane causes the first response. In each case, the change in fluorescence increases to a maximum over the 2 second analyte introductory period and then the signal slowly returns to its baseline value as the analyte gasses diffuse out of the detector.

Figure 7 shows similar temporal response curves for a Nile Red/PDMS sensor on an SU-8 device. Here, the analytes are again introduced to the sensor in a 2 second pulse and the fluorescence analysed using both the 580 and $640 \mathrm{~nm}$ filters. The sensor first detects methanol, then hexane and finally butan-2-ol, and the plots of the fluorescent intensity for both analysed wavelengths follow a similar shape. However, the plot of the fluorescence using the $580 \mathrm{~nm}$ filter shows a decrease in fluorescence opposite to that found using the Nile Red/PDMS sensor in the MSG device, where an increase is observed.

The peak maximum and the recovery time to $50 \%$ of peak maximum for the sensors on each of the devices at $580 \mathrm{~nm}$, are tabulated in Table 1. Comparison of the results for the Nile Red/PDMS sensors show that the response of the sensor on the MSG device was similar to that to the sensor on the SU-8 device for methanol but 3.5 times greater for butan-2-ol and 7 times greater for hexane. The recovery times of the sensors in the MSG device where up to $50 \%$ faster than those recorded for the SU-8 device. Comparison of the two different sensors on the MSG device reveals that Nile Red/PVP produces a superior sensor compared to Nile 
Red/PDMS for the detection of methanol, butan-2-ol and hexane, apart from a longer recovery time when detecting methanol.

Figure 8 shows the fluorescence changes of six sensors on a MSG device after an equilibrium state had been established. Positive values indicate an overall increase in fluorescent intensity of the dye/polymer complex in the presence of the analyte vapour, while negative values indicate an overall decrease. Figure 9 shows the fluorescent response of 4 sensors on a MSG device over 4 cycles of analyte introduction and purging. In this case, the fluorescent intensity is seen to decrease for the Nile Red/PVP sensor but increase for the other three sensors. Table 2 presents the maximum fluorescence change (\%) values for a 2 second methanol pulse using the data from figure 9. The standard deviation of the sensor response for each of the sensors is found to be less than $1 \%$.

\section{Discussion}

Figure 1 shows the structure of the dye used in these experiments, Nile Red. The large amount of conjugation in the structure means that the molecule has low energy, $\pi-\pi^{*}$ transition states. These allow electronic transitions to occur which lead to fluorescent light emission from the dye molecule. The rigidity of the extended carbon ring structure, as well as the presence of heterocycles, serves to increase the amount of fluorescence produced when compared to a simple heterocycle. This molecular rigidity enhances the quantum efficiency of the fluorescence by reducing the amount of radiationless deactivation occurring via structural rearrangement. A similar effect has also been seen to occur upon adsorbing a fluorescent dye to a solid surface [20]. The presence of the heteroatom within the extended conjugated structure has the effect of reducing the amount of non-luminescent transitions (via conversion to a triplet state) that occur in simple heterocyclic systems [21]. Consequently, Nile Red dye has been described as displaying positive solvatochromism 
leading to a bathochromic shift of $128 \mathrm{~nm}$ upon measuring the UV/vis/near IR spectra in hexane (a non-polar solvent) and water (a polar solvent) [22]. These effects serve to make Nile Red an ideal luminescent dye for use in an optical sensing system [23-24].

The polymers used in the sensing devices upon which the dye is immobilised function as a sorbent phase, attracting and concentrating analyte vapours when present. However, they also allow the vapours to diffuse out of the polymer matrices easily, providing a reversible response. The sensitivity and selectivity of each individual sensor is therefore controlled by the interaction between the Nile Red, the analyte and the host polymer matrix. However, one general disadvantage of using polymeric substrates is the difficulty in producing an evenly deposited film of uniform thickness. The SU-8 and MSG sensor devices, produced using microfabrication techniques, allow a uniform sensor surface to be defined within the detector.

Differences in the observed performance of the sensors on the MSG and SU-8 devices, derived from the temporal experiments (Figure 6 and 7), can be attributed to the surface area of the polymer deposited in the sensing layer. The honeycomb structure of the MSG device means that it has a larger surface area of polymer coating per unit area of the sensor compared to the SU-8 device. This means that the probability of interactions between the dye and the analyte vapours is higher in the MSG device sensors, leading to a greater change in the intensity of fluorescence produced per unit area of the sensor.

The performance of the individual polymers on the same device can be explained by looking at the possible physical interactions occurring between the analytes, the dye and the polymeric matrices. The hydrophobicity of the polymers used in the sensing elements and the molecular size and polarity of the analytes are particular indicators of possible sensor performance. A tentative assignment of these parameters is presented in table 3.

Compared to the PDMS sensor in the temporal response experiments, the greater fluorescence detected using the PVP sensor could result from the greater hydrophilicity of 
the PVP polymer. The high hydrophilicity means that the polymer will more readily form hydrogen bonds with the more polar alcohols rather than the relatively non-polar hexane. This would cause a higher fluorescence for the alcohols, as seen in figure 6(b), as the analyte molecule is held in close proximity to the polymer and the dye. The large response seen for the non-polar hexane in figure 7 also suggests that PDMS is more hydrophobic than PVP, as polar and non-polar species tend to attract other molecules of similar polarity.

Although the recovery time to $50 \%$ of peak fluorescent intensity for hexane is slower than that for the alcohols (Table 1), the response transients for the polar analytes do not recover to their baseline values as fast as that for hexane (Figure 6(b)). In comparison, the curves for the PDMS all recover their baseline values within the time period of the experiment (Figure 6(a)). Hydrogen bonding between the polar analytes and the polar PVP may cause the polar analytes to be held in the vicinity of the PVP sensor longer than the PDMS sensor.

Another factor in the relative strengths of the detected fluorescence observed for the Nile Red/PVP and Nile Red/PDMS sensors, in the presence of the analytes, could be the density of the deposited polymer film. Figure 6 shows that methanol produces a much more intense fluorescence with the PDMS film compared to the other two analytes, whereas all the analytes produce similar amounts of fluorescence with the PVP film. Due to the bulky pyrollidone group attached to the carbon backbone it can be assumed that the PVP film is less densely packed than that of the PDMS. This would allow all the analytes to penetrate the PVP polymer matrix to a similar extent, leading to a similar amount of fluorescence. The relatively low density of the matrix would also allow the analytes to diffuse into and out of the polymer quickly leading to low recovery times for the sensor. In contrast, the PDMS may be too dense to allow anything other than the methanol to penetrate easily, causing the other analyte vapours to be restricted to the polymer surface. In this case, the concentration of 
analyte at the surface will be lower than that in the polymer matrix, leading to a reduced number of interactions with the Nile Red/polymer and hence less fluorescence.

Figure 8 shows that the Nile Red/polymer sensors detect a decrease or an increase in fluorescence depending on the wavelength under study. Interestingly, in most of those results where there is a change in the measured fluorescence, from a decrease in fluorescence to an increase, the decrease in fluorescence always occurs at the lower wavelength studied, i.e. $580 \mathrm{~nm}$. Exceptions to this observation include the Nile Red/PDMS/ethanol and the Nile Red/PABS/ethanol complexes where the fluorescence at both wavelengths decreases and increases respectively, and the Nile Red/PDMS/hexane complex where changes in fluorescence are reversed. The differences in these results can be attributed to the positions of the absorption and emission spectra for each of the Nile Red/polymer/analyte complexes.

Figure 10 shows theoretical fluorescence absorption and emission spectra for a Nile Red/polymer/analyte complex based on an experimental emission curve for Nile Red dissolved in Dioxane solvent [16]. The absorption spectrum is at a maximum at wavelengths outside of those of the emission spectrum and, depending on the position of the spectra, the wavelengths analysed in the equilibrium state experiments will fall within one or other of the spectra, or both. For example, for the Nile Red/PDMS/ethanol complex both the analysis wavelengths fall within the absorption spectrum. For other complexes, one analysis wavelength falls within the emission wavelengths while the second falls within the absorbing wavelengths or vice versa.

\section{Conclusions}

Optical sensors based on the solvatochromic dye, Nile Red, immobilised within various supporting polymers, have been shown to detect simple organic vapours via fluorescence of the dye upon excitation by $533 \mathrm{~nm}$ optical radiation. MSG and SU-8 based devices 
containing the polymer/dye sensors have been used to produce fluorescence from the dye in the presence of analytes, such as hexane, methanol and butan-2-ol, and their performances compared. The fluorescence response, detected via an epifluorescence microscope and imaging CCD camera, shows that the MSG devices are superior to their SU-8 analogues, with respect to sensor response and recovery, in detecting these analytes.

These results can be attributed to the physical properties of, and the interactions between, the polymer/dye complex and the analytes, as well as the structure of the support for the sensors. By taking advantage of emission shift phenomenon of the solvatochromic dye in environments with different polarities, similar to the bathochromic shift seen for the dye within different solvent solutions [25], the responses of the sensors at two different wavelengths show potential for gas identification and discrimination.

\section{Acknowledgements}

The authors wish to thank the Overseas Research Students Awards (ORS, UK) and the University of Glasgow for supporting this work with the award of a grant to one of us (DL). Thanks are also due to Dr. N. Klauke, Dr. L. Cui and Dr. A. Glidle at the Department of Electronics and Electrical Engineering, University of Glasgow, for their assistance during the completion of this work. 


\section{References}

[1] J. W. Grate, S. J. Patrash, S. N. Kaganove, M. H. Abraham, B. M. Wise, N. B. Gallagher, Inverse least-squares modelling of vapour descriptors using polymer-coated surface acoustic wave sensor array responses, Anal. Chem. 73 (2001) 5247-5259.

[2] J. W. Grate, Acoustic wave microsensor arrays for vapour sensing, Chem. Rev. 100 (2000) 2627-2647.

[3] J. W. Grate, S. J. Patrash, M. H. Abraham, C. M. Du, Selective vapour sorption by polymers and cavitands on acoustic wave sensors: is this molecular recognition?, Anal. Chem. 68 (1996) 913-917.

[4] Q. Fang, D. G. Chetwynd, J. A. Covington, C. S. Toh, J. W. Gardner, Micro-gas-sensor with conducting polymers, Sens. Actuators, B, Chem. 84 (2002) 66-71.

[5] L. Cui, M. J. Swann, A. Glidle, J. R. Barker, J. M. Cooper, Odour mapping using microresistor and piezo-electric sensor pairs, Sens. Actuators, B, Chem. 66 (2000) 94-97.

[6] J. W. Gardner and P. N. Bartlett, Application of conducting polymer technology in microsystems, Sens. Actuators, A, Phys. 51 (1995) 57-66.

[7] J. W. Gardner, M. Craven, C. Dow, E. L. Hines, The prediction of bacteria type and culture growth phase by an electronic nose with a multi-layer perception network, Meas. Sci. Technol. 9 (1998) 120-127.

[8] M. E. Koscho, R. H. Grubbs, N. S. Lewis, Properties of vapour detector arrays formed through plasticization of carbon black-organic polymer composites, Anal. Chem., 74 (2002) $1307-1315$.

[9] B. J. Doleman, M. C. Lonergan, E. J. Severin, T. P. Vaid, N. S. Lewis, Quantitative study of the resolving power of arrays of carbon black-polymer composites in various vapoursensing tasks, Anal. Chem. 70 (1998) 4177-4190. 
[10] E. J. Severin, R. D. Sanner, B. J. Doleman, N. S. Lewis, Differential detection of enantiomeric gaseous analytes using carbon black-chiral polymer composites, chemically sensitive resistors, Anal. Chem. 70 (1998) 1440-1443.

[11] K. J. Albert and D. R. Walt, High-speed fluorescence detection of explosives-like vapours, Anal. Chem.71 (2000) 1947-1955.

[12] T. A. Dickinson, K. L. Michael, J. S. Kauer, D. R. Walt, Convergent, self-encoded bead sensor arrays in the design of an artificial nose, Anal. Chem. 71 (1999) 2192-2198.

[13] K. J. Albert, N. S. Lewis, C. L. Schauer, G. A. Sotzing, S. E. Stitzel, T. P. Vaid, D. R. Walt, Cross-reactive chemical sensor arrays, Chem. Rev. 100 (2000) 2595-2626.

[14] N. A. Rakow and K. S. Suslick, A colorimetric sensor array for odour visualization, Nature 406 (2000) 710-713.

[15] J. White, J. S. Kauer, T. A. Dickinson, D. R. Walt, Rapid analyte recognition in a device based on optical sensors and the olfactory system, Anal. Chem. 68 (1996) 2191-2202.

[16] K. J. Albert, D. R. Walt, D. S. Gill, T. C. Pearce, Optical multibead arrays for simple and complex odour discrimination, Anal. Chem. 73 (2001) 2501-2508.

[17] J. White, J. S. Kauer, T. A. Dickinson and D. R. Walt, Rapid analyte recognition in a device based on optical sensors and the olfactory system, Anal. Chem. 68 (1996) 2191-2202.

[18] S. M. Dickinson, J. White, J. S. Kauer and D. R. Walt, A chemical-detecting system based on a cross-reactive optical sensor array, Nature 382 (1996) 697-700

[19] V. Beniot, A. Steel, M. Torres, Y-Y. Yu, H. Yang and J. Cooper, Evaluation of threedimensional microchannel glass biochips for multiplexed nucleic acid fluorescence hybridization assays, Anal. Chem. 73 (2001) 2412-2420.

[20] R. J. Hurtubise, Solid surface luminescence spectroscopy, Anal. Chem. 61 (1989) 889A-895A.

[21] D. A. Skoog and J. J. Leary, Principles of Instrumental Analysis, Saunders College Publishing, Florida, $4^{\text {th }}$ edn., 1992. 
[22] C. Reichardt, Solvatochromic dyes as solvent polarity indicators, Chem. Rev. 94 (1994) 2319-2358.

[23] I. Levitsky, S. G. Krivoshlykov, and J. W. Grate, Rational design of a Nile Red/polymer composite film for fluorescent sensing of organophosphate using hydrogen bond acidic polymers, Anal. Chem. 73 (2001) 3441-3448.

[24] T. A. Dickinson, J. White, J. S. Kauer and D. R. Walt, Current trends in artificial nose technology, Trends. Biotechnol. 16 (1998) 250-258.

[25] H. Du, R. A. Fuh, J. Li, A. Corkan, J. S. Lindsey, PhotochemCAD: A computer aided design and research tool in photochemistry, Photochemistry and Photobiology 68 (1998) 141-142. 


\section{Figure captions}

Figure 1. Chemical structure of the polymers used as the support matrices for (a) the solvatochromic dye, Nile Red. The polymers are, (b) poly(dimethylsiloxane) (PDMS), (c) poly(styrene) (PS), (d) poly(methylmethacrylate) (PMMA), (e) poly(caprolactone) (PC), (f) poly(vinylpyrrolidone) (PVP), and (g) poly(acrylonitrile-co-butadiene-co-styrene) (PABS).

Figure 2. SEM micrograph of the microstructure of a MSG bundle showing the honeycomb arrangement of the etched glass. The dye/polymer coated MSG is to the bottom right of the image whereas the darker holes to the top left are uncoated MSG. Inset is a cross-sectional diagram of the bundle structure $(\mathrm{A}=50 \mu \mathrm{m}, \mathrm{B}=0.5 \mathrm{~mm})$.

Figure 3. (a) SEM micrograph of the centre of an SU-8-based sensor device, prior to addition of the glass top plate, showing the etched channels for the dye/polymer sensors (channel dimensions: $50 \mu \mathrm{m}$ wide, $200 \mu \mathrm{m}$ deep). Inset is an overhead optical image of the same area incorporating eight Nile Red/polymer combinations within the channels $(\mathrm{Bar}=50$ $\mu \mathrm{m})$. (b) Cross-sectional diagram of the device structure.

Figure 4. Schematic diagram of the imaging epifluorescence microscope incorporating a monochromator to define the excitation frequency of $533 \mathrm{~nm}$ and two emission filters, housed within the filter wheel, for analysis of the emitted fluorescence at 580 and $640 \mathrm{~nm}$ (band-pass $10 \mathrm{~nm}$ ).

Figure 5. Schematic diagram of the bespoke gas-flow system, incorporating a 5-port electric valve, used to introduce the analyte gasses to the sensors at a rate of $0.4 \mathrm{~L} \mathrm{~min}^{-1}$. 
Figure 6. Fluorescent temporal response of sensors on a MSG device to 2-s vapor pulses of (O) butan-2-ol, ( $\nabla)$ hexane and $(\square)$ methanol using the $580 \mathrm{~nm}$ filter. The response of (a) Nile Red/PDMS and (b) Nile Red/PVP sensors were recorded using 0.15 second intervals between readings.

Figure 7. Fluorescent temporal response of the Nile Red/PDMS sensor contained within an SU-8 device to (O) butan-2-ol, $(\nabla)$ hexane and $(\square)$ methanol using (a) the $580 \mathrm{~nm}$ filter and (b) the $640 \mathrm{~nm}$ filter. The interval between measurements was 0.15 seconds.

Figure 8. Fluorescence change recorded at 580 and $640 \mathrm{~nm}$ for analyte vapours, (a) ethanol and (b) hexane, in an equilibrium state using a MSG device.

Figure 9. Sensor signal repeatability and recovery for 4 polymer/dye sensors upon introducing a $2 \mathrm{~s}$ methanol pulse, followed by a $20 \mathrm{~s}$ nitrogen purge. The response of (O) Nile Red/PS, $(\nabla)$ Nile Red/PC, $(\square)$ Nile Red/PVP and $(\diamond)$ Nile Red/PMMA sensors on a MSG device are presented, analysed using the $640 \mathrm{~nm}$ filter with measurements taken at 0.15 second intervals.

Figure 10. Normalised absorption (dashed line) and emission (solid line) spectra for a theoretical NR/polymer/analyte complex. The plot shows possible absorbance at $580 \mathrm{~nm}$ and emission at $640 \mathrm{~nm}$, causing a decrease and an increase in the detected fluorescence at each wavelength respectively, upon introduction of the analyte to the system. 


\section{Table captions}

Table 1 Percentage fluorescent intensity changes and recovery time to $50 \%$ of signal maximum for NR/PVP sensors on MSG and SU-8/glass devices using the $580 \mathrm{~nm}$ filter.

Table 2 Average maximum fluorescence intensity change for polymer/dye sensors in a MSG sensor subjected to a 2 second pulse of methanol vapour.

Table 3 Sensing polymer hydrophobicity and analyte molecular size and polarity. 


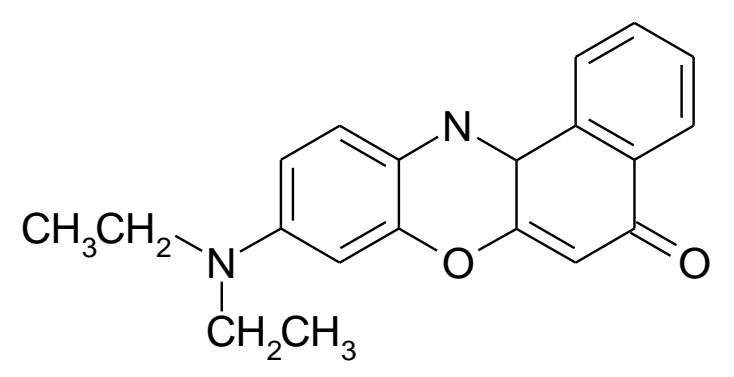

(a)<smiles>CC(C(C)(C)C)C(C)(C)c1ccccc1</smiles>

(c)<smiles>CC(C)(C)OCCCCCC(=O)C(F)(F)F</smiles>

(e)<smiles>[3H]C([3H])O[Si](C)(C)C</smiles>

(b)<smiles>CCC(C)(C)C(=O)OC</smiles>

(d)<smiles>CC(C)(C)CC(N1CCCC1=O)C(C)(C)C</smiles>

(f)

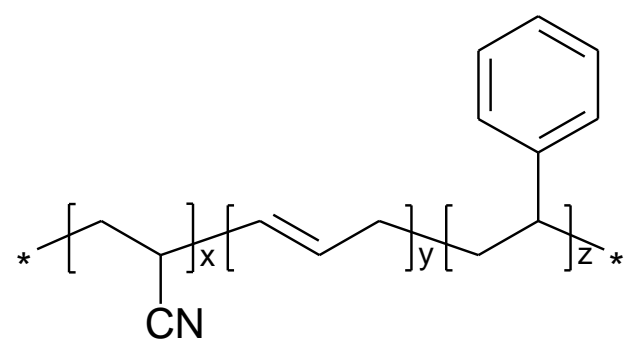

(g) 
Figure 2

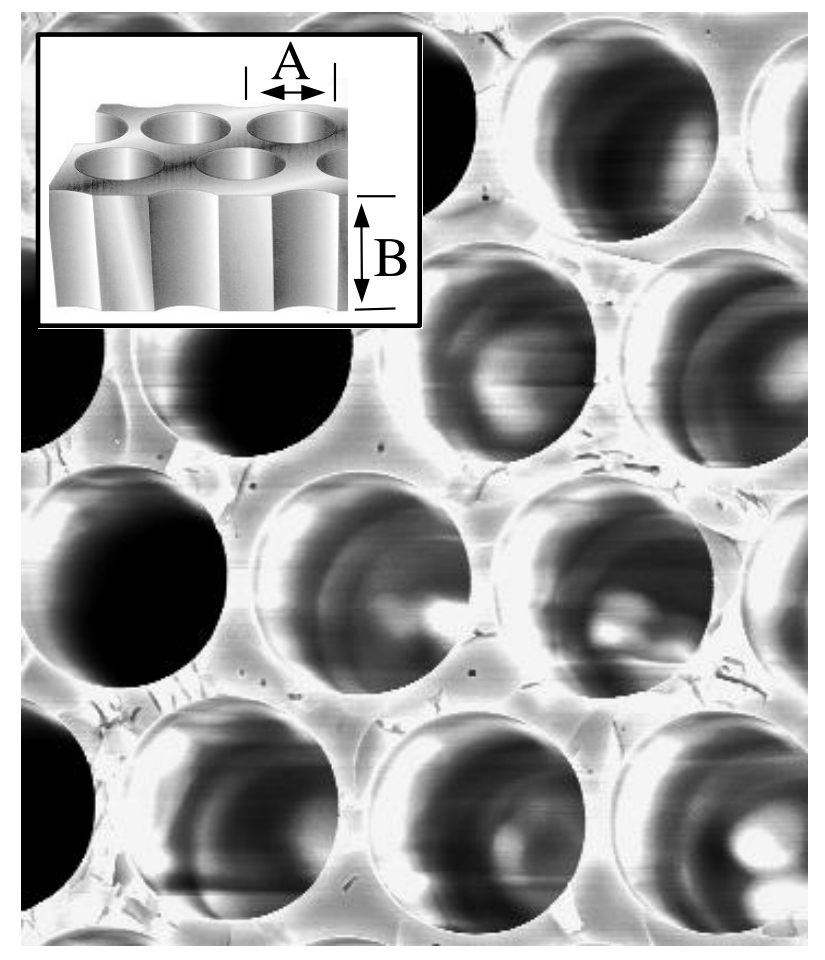




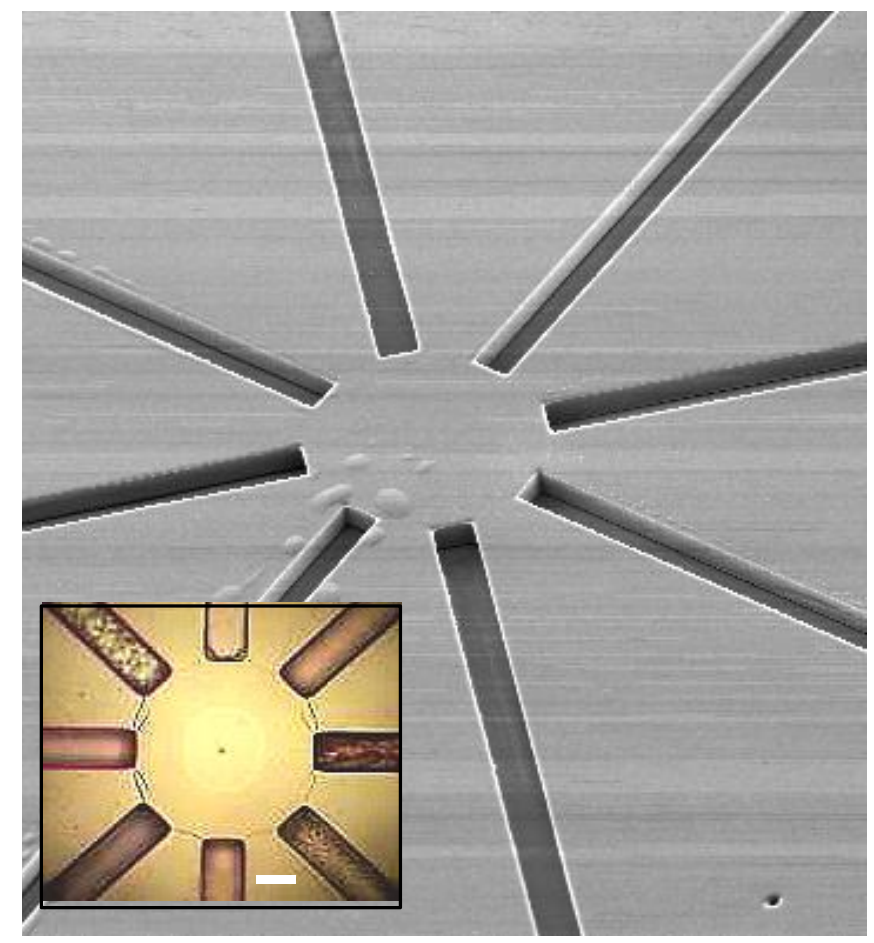

(a)

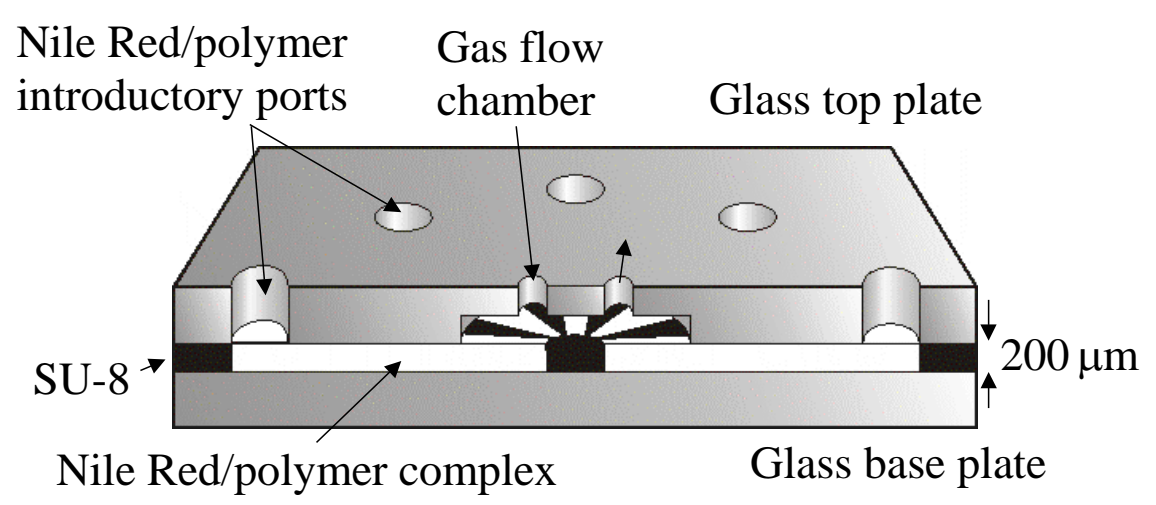

(b) 


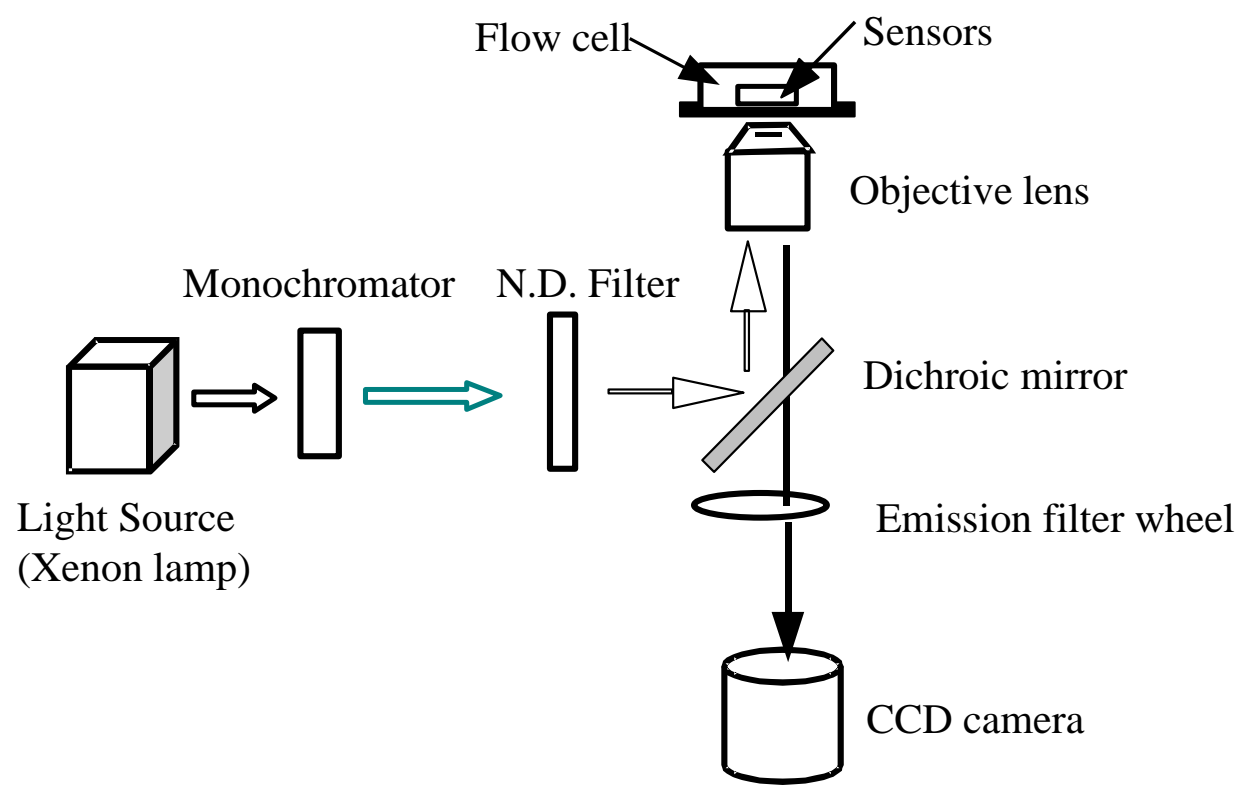




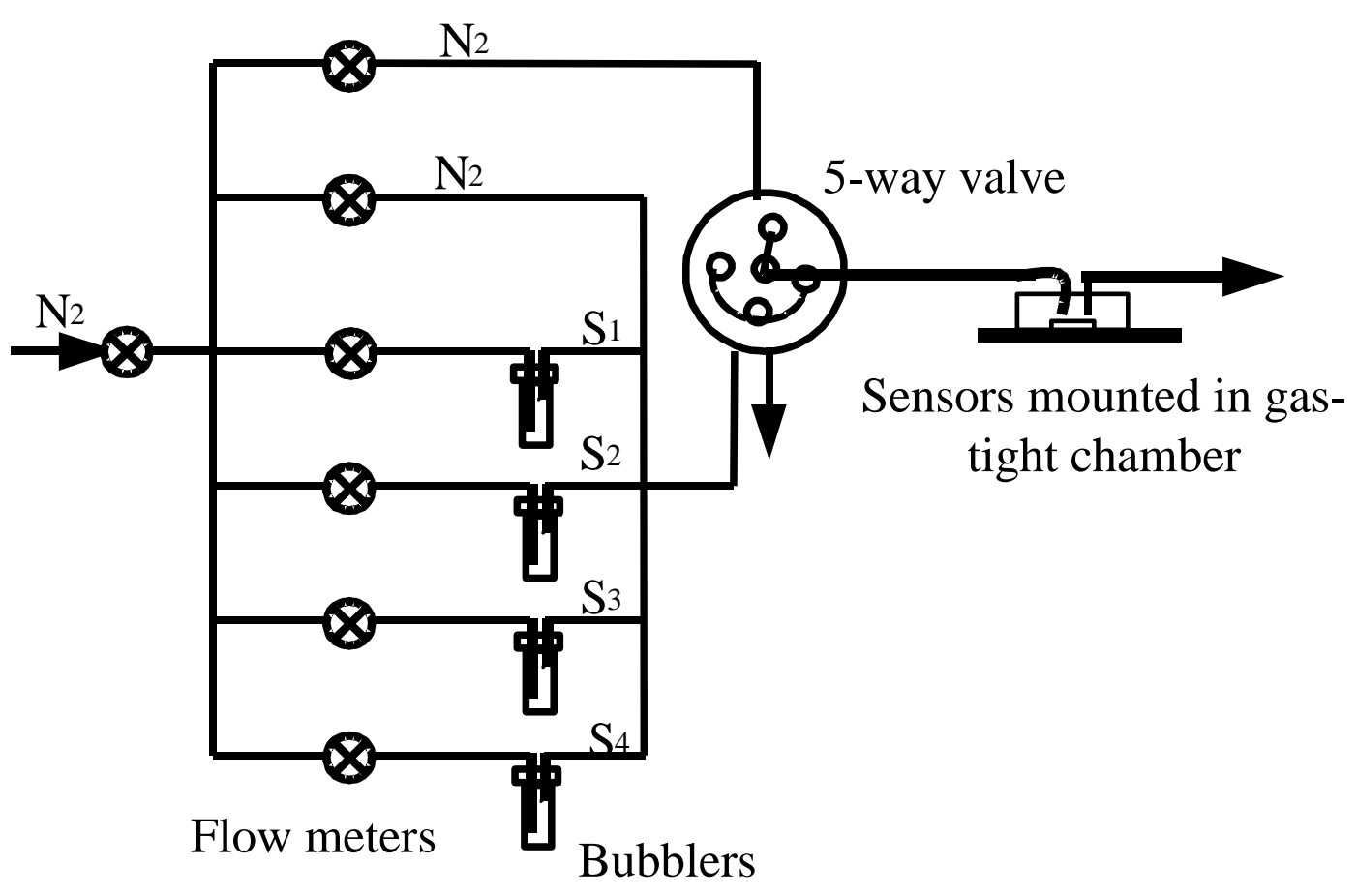



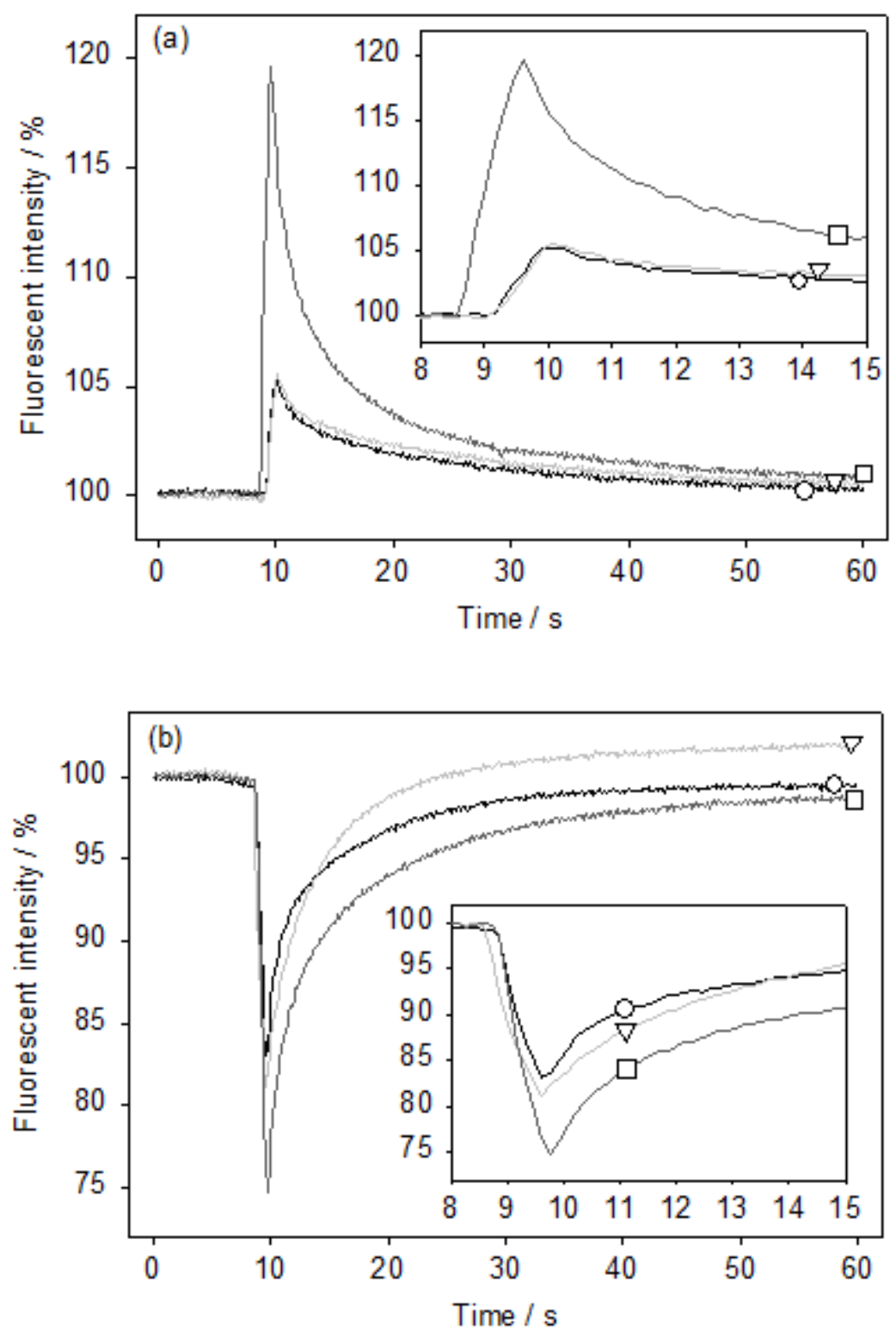

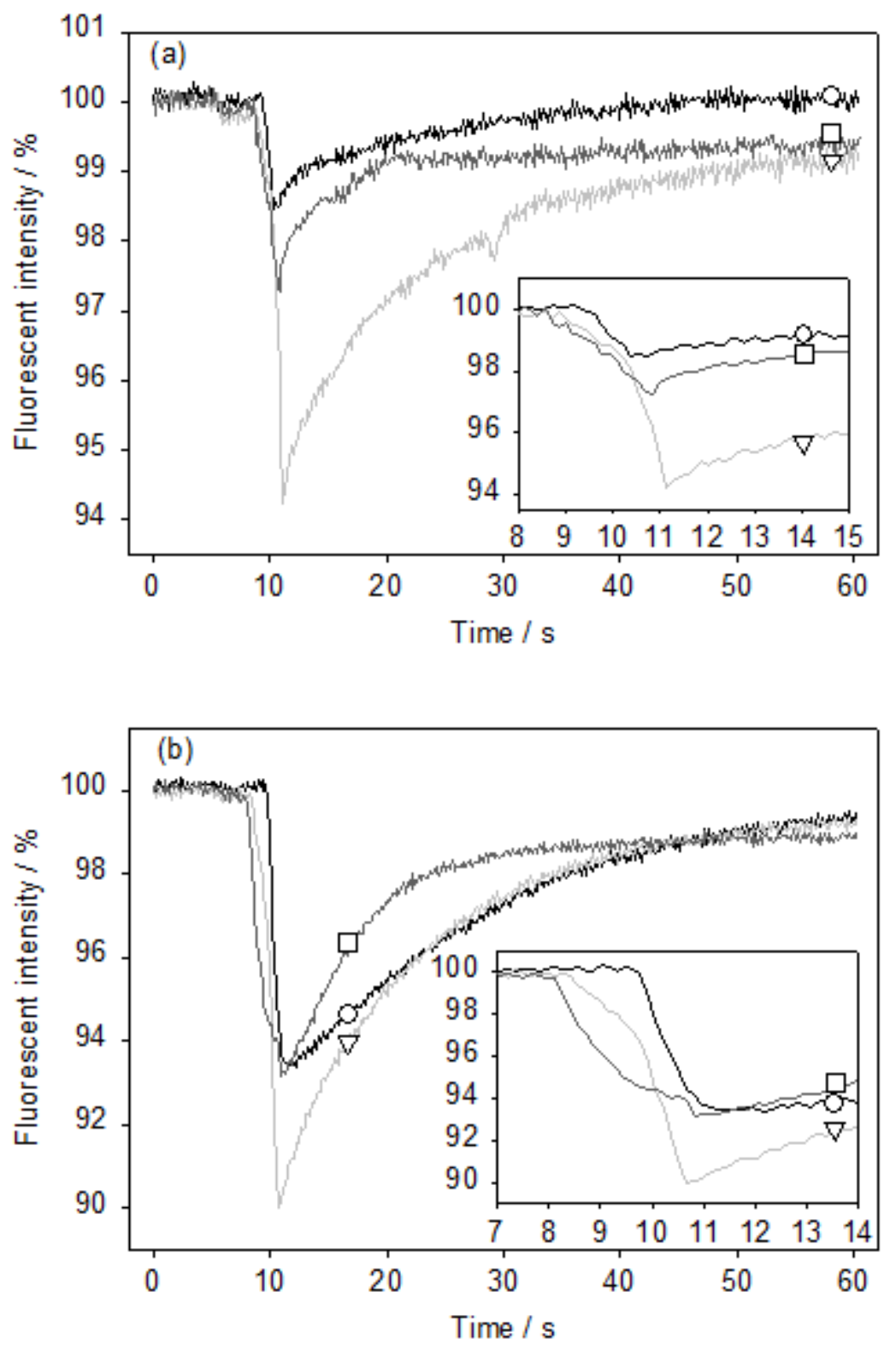

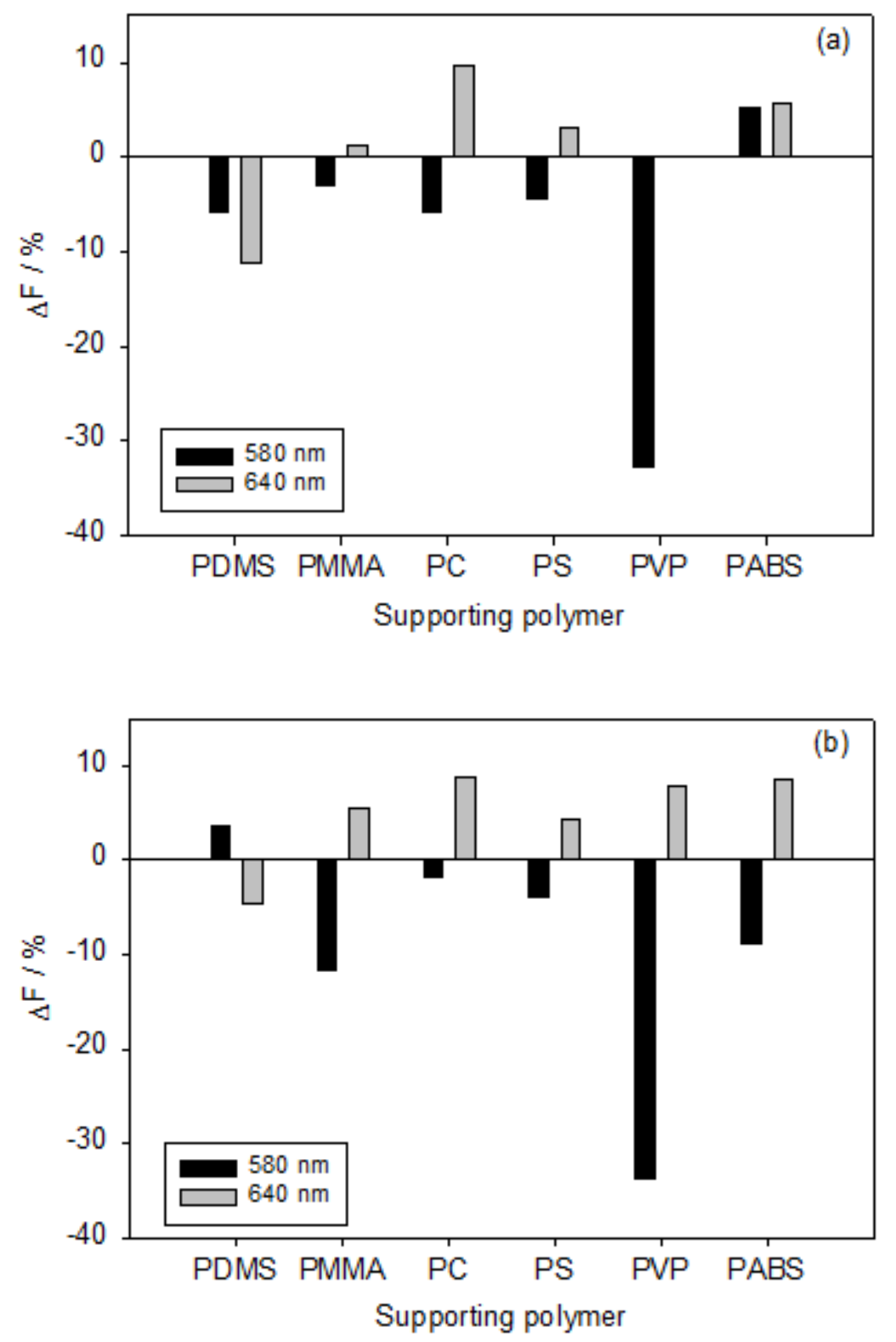


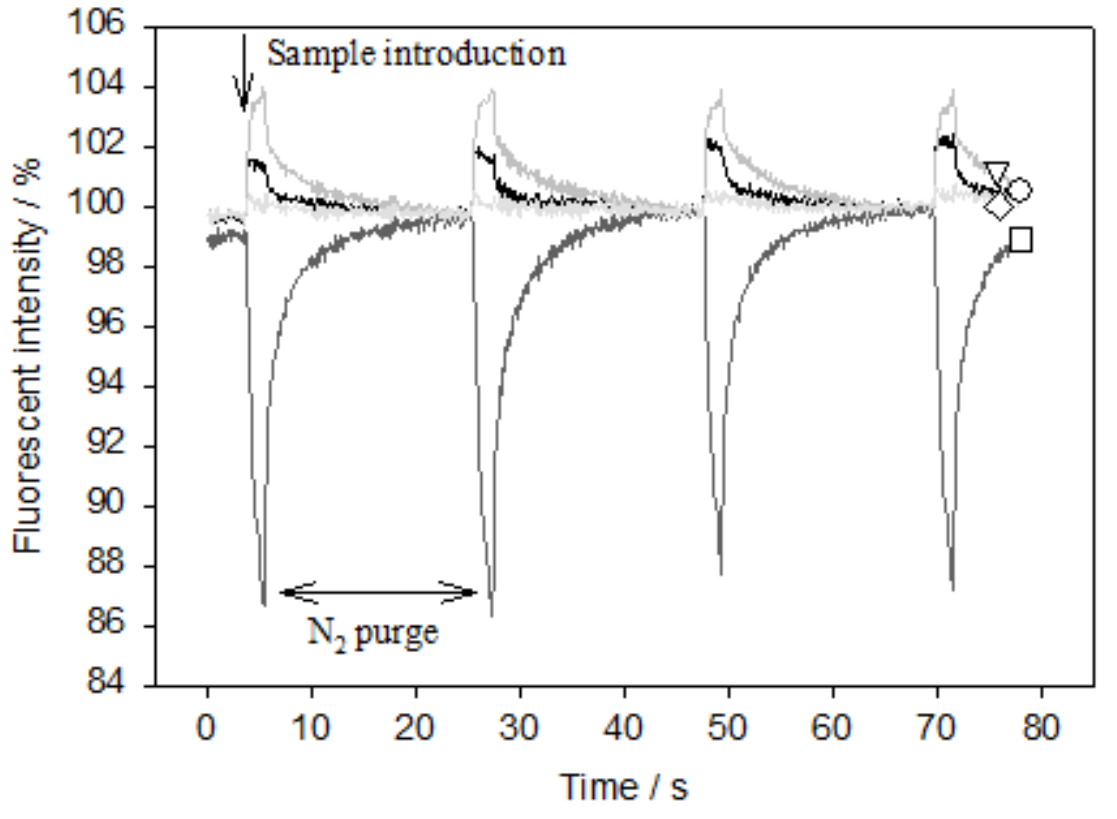




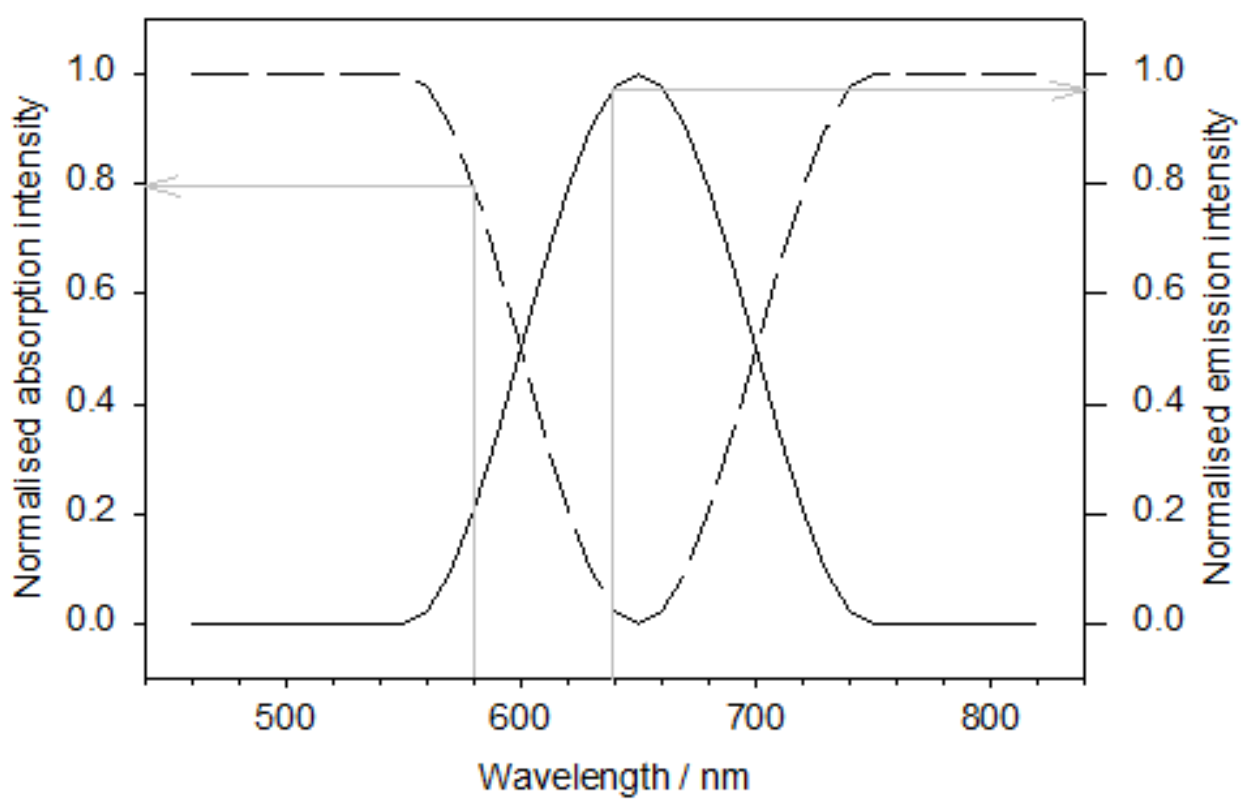




\begin{tabular}{|c|c|c|c|c|c|c|}
\hline \multirow[t]{2}{*}{ Device / Sensor } & \multicolumn{3}{|c|}{$\begin{array}{c}\text { Maximum fluorescent intensity } \\
\text { change }(\Delta \mathbf{F}) / \%\end{array}$} & \multicolumn{3}{|c|}{$50 \%$ signal recovery time / s } \\
\hline & Butan-2-ol & Hexane & Methanol & Butan-2-ol & Hexane & Methanol \\
\hline MSG / PDMS & 5.28 & 19.63 & 5.55 & 4.80 & 6.15 & 2.10 \\
\hline MSG / PVP & 16.89 & 25.25 & 18.89 & 2.10 & 2.70 & 2.40 \\
\hline SU-8 / PDMS & 1.55 & 2.74 & 5.78 & 5.98 & 9.13 & 4.47 \\
\hline
\end{tabular}




\begin{tabular}{lc}
\hline Sensor & $\begin{array}{c}\text { Average maximum fluorescence } \\
\text { intensity change }(\Delta \mathbf{F}) / \%\end{array}$ \\
\hline NR / PMMA & $0.51 \pm 0.20$ \\
NR / PC & $3.91 \pm 0.29$ \\
NR / PS & $2.04 \pm 0.36$ \\
NR / PVP & $-12.98 \pm 0.60$ \\
\hline
\end{tabular}




\begin{tabular}{ccc}
\hline Polymer hydrophobicity & Analyte polarity & Analyte molecular size \\
\hline Hydrophobic & Most polar & Largest \\
PS & Methanol & Butan-2-ol \\
PDMS & Butan-2-ol & Hexane \\
PMMA & Hexane & Methanol \\
PABS & Least polar & Smallest \\
PC & \\
PVP & \\
Hydrophilic & \\
\hline
\end{tabular}

